\title{
Percutaneous treatment with the Örmeci technique of cystic echinococcosis: experience of single center
}

\begin{abstract}
Background: Hydatid disease is caused by the larval stage of Echinococcus Granulosis which is an important public health and economic problem. The treatment of hydatid cyst is not optimal yet.

Objective: We want to show the Ormeci technique is the one of the best treatment options of hydatid cyst.

Materials and methods: Five hundred thirty-seven patients with seven hundred and sixteen hydatid cysts had been treated by percutaneous way using the Ormeci technique since 1991. The percutaneous puncture was performed under sonographic guidance using a 22-gauge Chiba needle as a one-step procedure in CE type 1 and $3 \mathrm{~A}$. However, two to six Chiba needles were used in different locations at the same time in CE type $2 b, 2 c$ and 3 B cysts. The mean follow-up time was $51.24 \pm 62.99$ (minimum 3.00, maximum 340.00) months.
\end{abstract}

Results: This technique increased the cure rate of CE type 2 and $3 B$ hydatid cysts up to $91.7 \%$ without recurrences. The success rate for all types of cysts treated was $95 \%$ without mortality. The technique had less morbidity $(8.86 \%)$ without fistula and abscess.

Conclusion: Ormeci technique can be safely and successfully used for the treatment of all kinds of active hydatid cysts.

Keywords: PAIR, ormeci technique, hydatid cyst
Volume II Issue 6 - 2020

\author{
Necati Örmeci,', Ozgun Omer Asiller,2 \\ Cagdas Kalkan, ${ }^{3}$ Zeynep Ellik, ${ }^{2}$ Aysun \\ Caliskan Kartal, Ramazan Erdem Er, ${ }^{2}$ Atilla \\ Halil Elhan, ${ }^{4}$ Tugrul Örmeci ${ }^{5}$ \\ 'Department of Internal Medicine, Istanbul Health and \\ Technology University, Turkey \\ ${ }^{2}$ Department of Gastroenterology, Ankara University, Turkey \\ ${ }^{3}$ Ministry of Health, Ankara City Hospital,Ankara, Turkey \\ ${ }^{4}$ Department of Medical Statistics, Ankara University, Turkey \\ ${ }^{5}$ Department of Radiology, Medipol University, Turkey
}

Correspondence: Necati Ormeci, Department of Internal Medicine, Division of Gastroenterology, Istanbul Health and Technology University, School of Medicine, Istanbul, Turkey, Tel +90 (532) 27358 98, Email normeci@yahoo.com

Received: November 06, 2020 | Published: December 10, 2020
Abbreviations: EG, echinococcus granulosis; CE, cystic echinococcosis; PAIR, puncture aspiration injection re-aspiration; HC, hydatid cysts; DPAI, double puncture aspiration injection; MoCAT, modified catheter technique; ABZ, Albendazole

\section{Introduction}

Hydatid disease is caused by the larval stage of Echinococcus Granulosis (EG) which threatens human life by compressing vital organs and rupturing into the peritoneal, pleural spaces or biliary ducts. It also becomes a heavy economic burden on the people by costing 285,407 Disability Adjusted Life Years (DALYs) or approximately 193,529.740 US Dollars annually. ${ }^{1}$ Almost $70 \%$ of Cystic Echinococcosis (CE) is in the liver. However, if it passes through systemic circulation, it can be observed in any organ such as the lung, kidney, spleen, muscles, brain etc. Although CE has been a well-known disease since Hippocrates, this disease does not have an optimal treatment yet. Small cystic lesions that are asymptomatic and less than $4 \mathrm{~cm}$ in diameter can be periodically followed every three or six months. However, all symptomatic and alive CEs /CE1, CE2, CE3A and CE3B should be treated. Surgical treatment was the gold standard of treatment until the last three decades. Medical treatment and Puncture Aspiration Injection Re-aspiration (PAIR) techniques became other options used in the treatment of hydatid diseases after the approval of Benzimidazole derivatives as potent, anti-helminthic drugs in the 1960s and puncturing and evacuating of hydatid cysts in 1985. The current study presented the 29years single-center experience of percutaneous treatment with the Ormeci Technique for CE.

\section{Methods}

Nine hundred and forty-two patients with one thousand three hundred and sixty-seven hydatid cysts had been diagnosed at Ankara University, School of Medicine, and Department of Gastroenterology since 1991. Five hundred thirty-seven patients with 716 hydatid cysts (HC) had been consecutively treated by the percutaneous method with the Ormeci technique to date. A hundred and fifty-four patients with 159 cysts which were treated by the percutaneous way were excluded from the study due to short follow-up time less than three months. Sixteen patients with $20 \mathrm{HCs}$ were excluded because of multiple hydatid cysts in other organs as well as the liver. Four hundred and seventy-four patients (303 females, 171 males) with $537 \mathrm{HCs}$ were included in this study. The mean age was $43.88 \pm 0.739$ years (youngest 5, oldest 90) (Figure 1). The HD was diagnosed using ultrasonography, $\mathrm{CT}$, MRI and hemagglutination inhibition test. Ultrasonographic diagnostic criteria were based on Gharbi's classification and WHO consensus reports. If we had difficulties in diagnosing the HD, we applied to the hemagglutination inhibition test, CT and/or MRI techniques.

\section{Criteria of inclusion}

All alive hydatid cysts type CE type 1 (Gharbi type 1), CE type 2 a, 2b, 2c (Gharbi type 3), CE type 3A (Gharbi type 3) and CE type 3B (Gharbi type 3) were included for the percutaneous treatment. All patients of any ages who had symptomatic diseases were included in the study. 


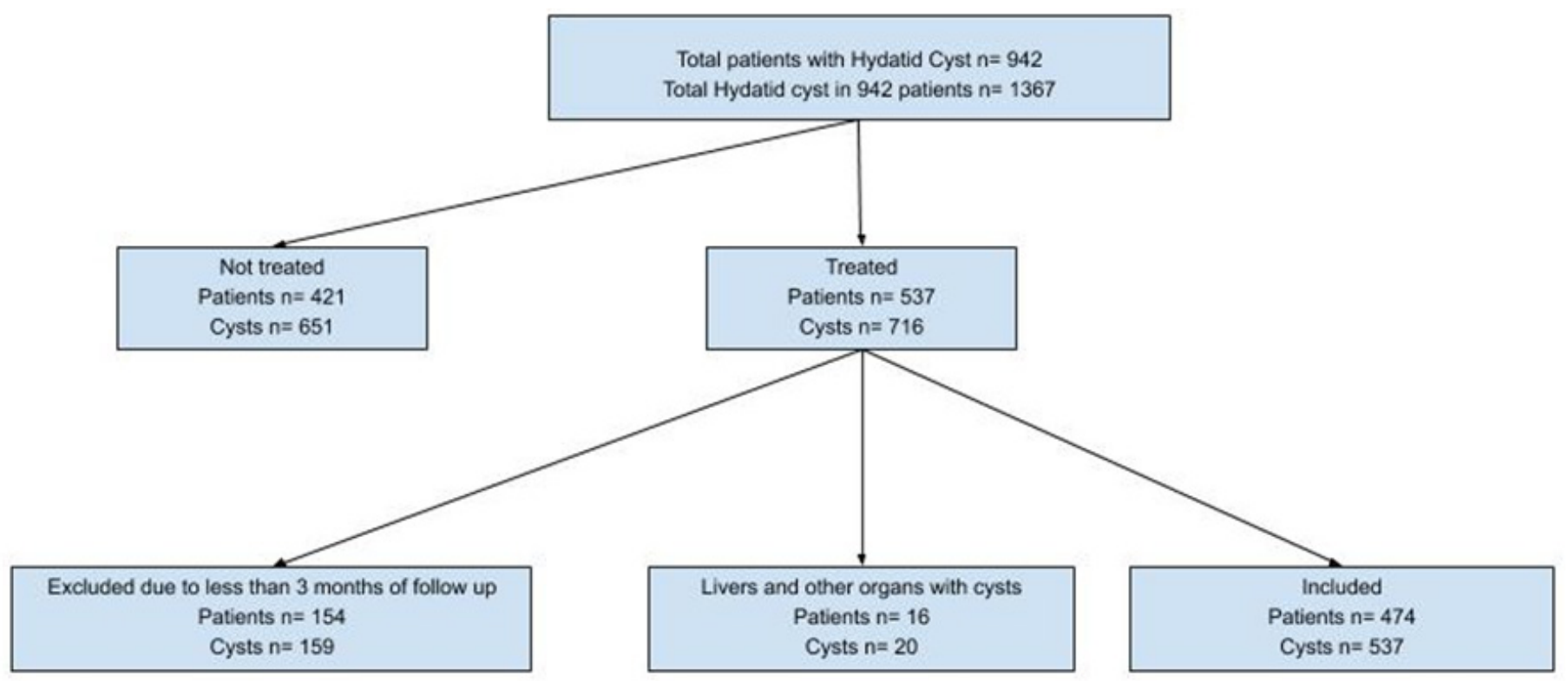

Figure I The flowchart of study.

\section{Criteria of exclusion}

All dead hydatid cysts CE type 4 and 5 were excluded from the study. The patients who had hemorrhagic disorder were excluded from the study.

All procedures were performed under sonographic guidance in the ultrasonography unit of a gastroenterology department that was fully equipped against an emergency condition. An intravenous line was established. The patients were positioned according to the location of the cysts as left lateral, right lateral or supine position. All patients were given $5 \mathrm{mg}$ of meperidine and $40 \mathrm{mg}$ methyl prednisolone as sedo-analgesia just before the procedure. The percutaneous puncture was performed under sonographic guidance using a 22-gauge Chiba needle as a one-step procedure in CE type 1 and $3 \mathrm{~A}$. However, two to six Chiba needles were used in different locations at the same time in the cyst of CE type 2, 3A and 3B (Figure 2). For every $1 \mathrm{~cm}$ of the long diameter of the cyst lesion, $3 \mathrm{cc}$ of fluid from the cysts was aspirated, which was almost the same amount of cc in volume for the CE type 1 or Gharbi type 1 and CE type 3A or Gharbi type 2 hydatid cysts. A $2 \mathrm{cc}$ of pure alcohol $(96 \%$ ) and $1 \mathrm{cc}$ of polidocanol 1\% (ethoxysclerol $1 \%$, Kreussler Pharma, Wiesbaden, Germany) were injected into the cysts right after the aspiration of CE type 1 and type 3A (Gharbi type 1 and 2), without the aspiration of CE type $2 \mathrm{a}, 2 \mathrm{~b} 2 \mathrm{c}$ and $3 \mathrm{~B}$ or Gharbi type 3, for each centimeter of the long diameter of the cysts. The polidocanol $(1 \%)$, had been used by us for the first time to close the connection among the cysts and blood vessels, lymphatic vessels and/or biliary ducts since 1991. The total amount of pure alcohol and polidocanol were injected equally among the CE type $2 a, 2 b, 2 c$ and type 3B (Gharbi type 3) cysts needles. We waited for five minutes for all scolexes to be killed and the needle/or needles were taken back. All patients were followed up for the function of vital organs for two to three hours and the patients were sent to their home. The patients were followed up one day, three months and six months after and each year by USG in terms of treatment criteria, blood checking and sometimes CT.

\section{Statistical analysis}

Descriptive statistics were summarized as counts and percentages for categorical variables, mean and standard deviations and median (minimum and maximum) for others. Categorical variables between groups were compared using the Chi-Square or Fisher's exact tests. The difference between the two dependent groups was evaluated with a paired $t$-test. Linear mixed-effects models were used to explain the variation in the outcome variable to clarify the clustered structure of the data. A $P$ value less than 0.05 was considered significant due to the nested structure of the data.

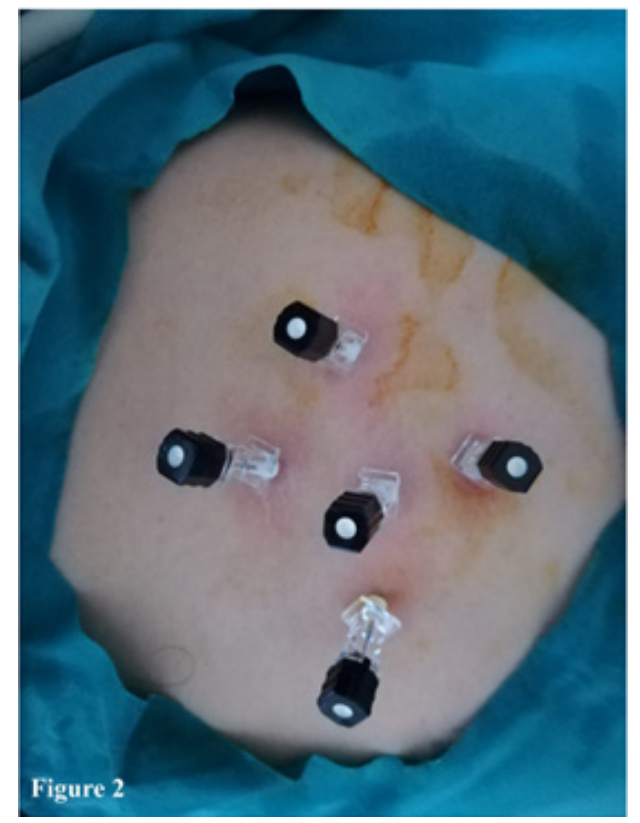

Figure 2 Multi-puncture for different localizations during the treatment of $\mathrm{CE}$ type $2 \mathrm{~B}, 2 \mathrm{C}$ and $3 \mathrm{~B}$ of hydatid disease. 


\section{Results}

Types of hydatid cysts which were approved in study were type 1-270, type 2-61, type 3-206 respectively. Pretreatment mean diameter of the cysts was $83.34 \pm 34.62 \mathrm{~cm}$ in diameter. Post treatment mean diameter of the cysts was $72.55 \pm 32.8 \mathrm{~cm}$ in diameter.

The patients were evaluated by detachment, perforating and color changing of the germinative membrane, reduction of cyst volume; degeneration, solidification and calcification of the cysts on the ultrasound examination during and one day after the procedure. The germinative membrane of some of the cysts $(46.7 \%)$ was detached from the cyst wall, became white, were torn apart and went to pieces down in the type of CE 1 and CE 3A (Figure 3) at the time of the treatment. The $\mathrm{CE} 2 \mathrm{~b}, 2 \mathrm{c}$ and $3 \mathrm{~B}$ cysts degenerated, changed color and some of the germinative membrane of daughter cysts were detached.
They became smaller $(87.7 \%)$ and $21(3.9 \%)$ of the cysts disappeared. They solidified $(91.0 \%)$ and calcified $(15.4 \%)$ during the followup (Figures 4). Interestingly, 47 cysts in 36 patients were affected by the treatment of another cyst. They resulted in degeneration and they finally died (Figure 5). Blood samples were collected to check $\mathrm{CBC}$, sedimentation rate, ALT; AST, alkaline phosphatase, GGT and bilirubin before and after the procedure for all patients. The mean follow-up time was $51.24 \pm 62.99$ (minimum 3.00, maximum 340.00) months. The treatment was effective. (Table 1) When the treatment was not efficient on the first puncture, a second $[\mathrm{n}=53(9.9 \%)]$, third $[\mathrm{n}=8(1.5 \%)]$ and fourth puncture $[\mathrm{n}=2(0.4 \%)]$ were applied during the follow-up. Forty-seven (9.91\%) out of 474 patients applied to us with several complications before the treatment due to HD. Of 474 patients, $42(8.86 \%)$ had some complications after the treatment. (Tables $2 \& 3$ )

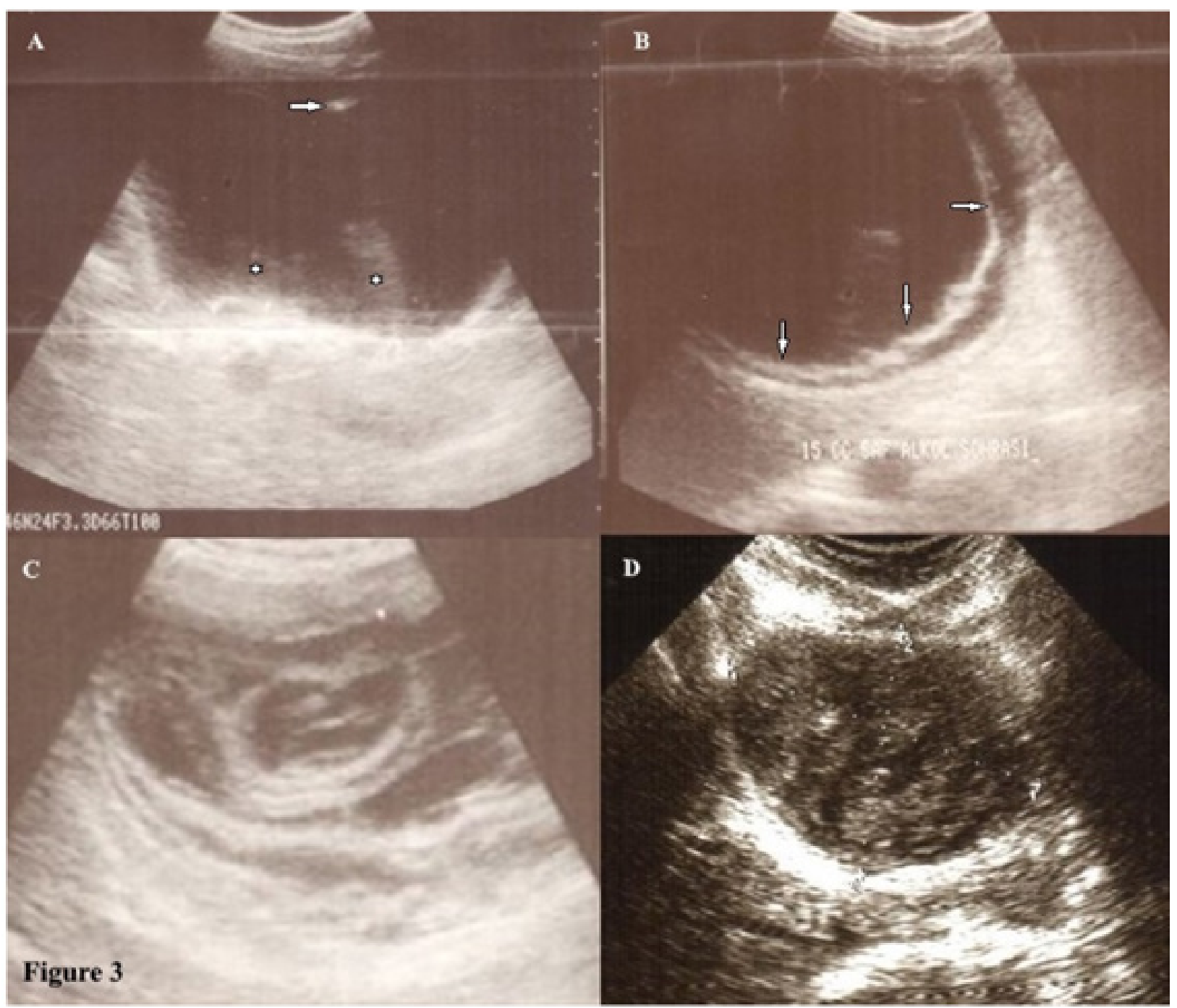

Figure 3 Eight-year-old girl with hydatid cyst (stage CE type I according to WHO classification).

(A) Before the percutaneous treatment, there is an anechoic cyst with fine internal echoes representing the 'hydatid sand' (asterisks), the arrow shows the needle tip.

(B) The germinative membrane (arrows) detached right after the treatment.

(C) The onionskin pattern is seen in the solidified hydatid cyst after a few months of treatment.

Absolutely solidified hydatid cyst (stage CE type 4 according to WHO classification) seen 27 years after the treatment. 


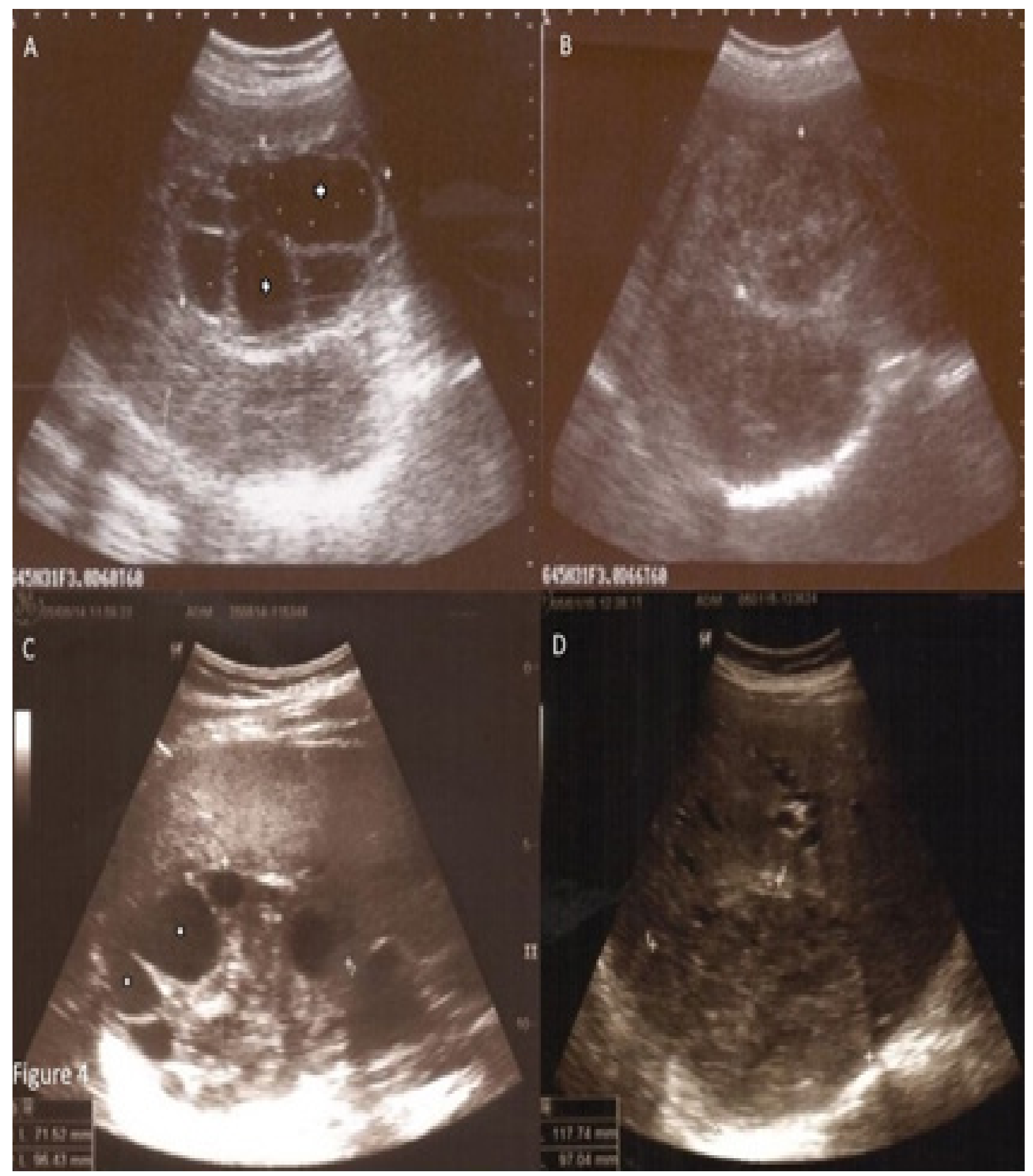

Figure 4 (A) A large multi-septated cystic lesion (stage CE Type 2 B according to WHO classification). Multipl daughter cysts (asterisks) are seen as large multiseptated cystic lesion in the right lobe of liver. It represents as 'honeycomb' aspect.

(B) After treatment, the cystic feature of the lesion has disappeared and it is observed in solid form.

(C) The daughter cysts within a solid matrix (stage CE Type 3 B according to WHO classification).

The heterogeneous lesion with cystic components is observed in the liver before treatment. The solid matrix is in centrally and daughter cysts (asterisks) in periphery of the lesion are found.

(D) After the treatment fluid content has totally disappeared. The lesion is seen as solid, like pseudotumor.

Citation: Örmeci N,Asiller OO, Kalkan C, et al. Percutaneous treatment with the Örmeci technique of cystic echinococcosis: experience of single center. Gastroenterol Hepatol Open Access. 2020; I (6):223-230. DOI: 10.15406/ghoa.2020.I I.00443 


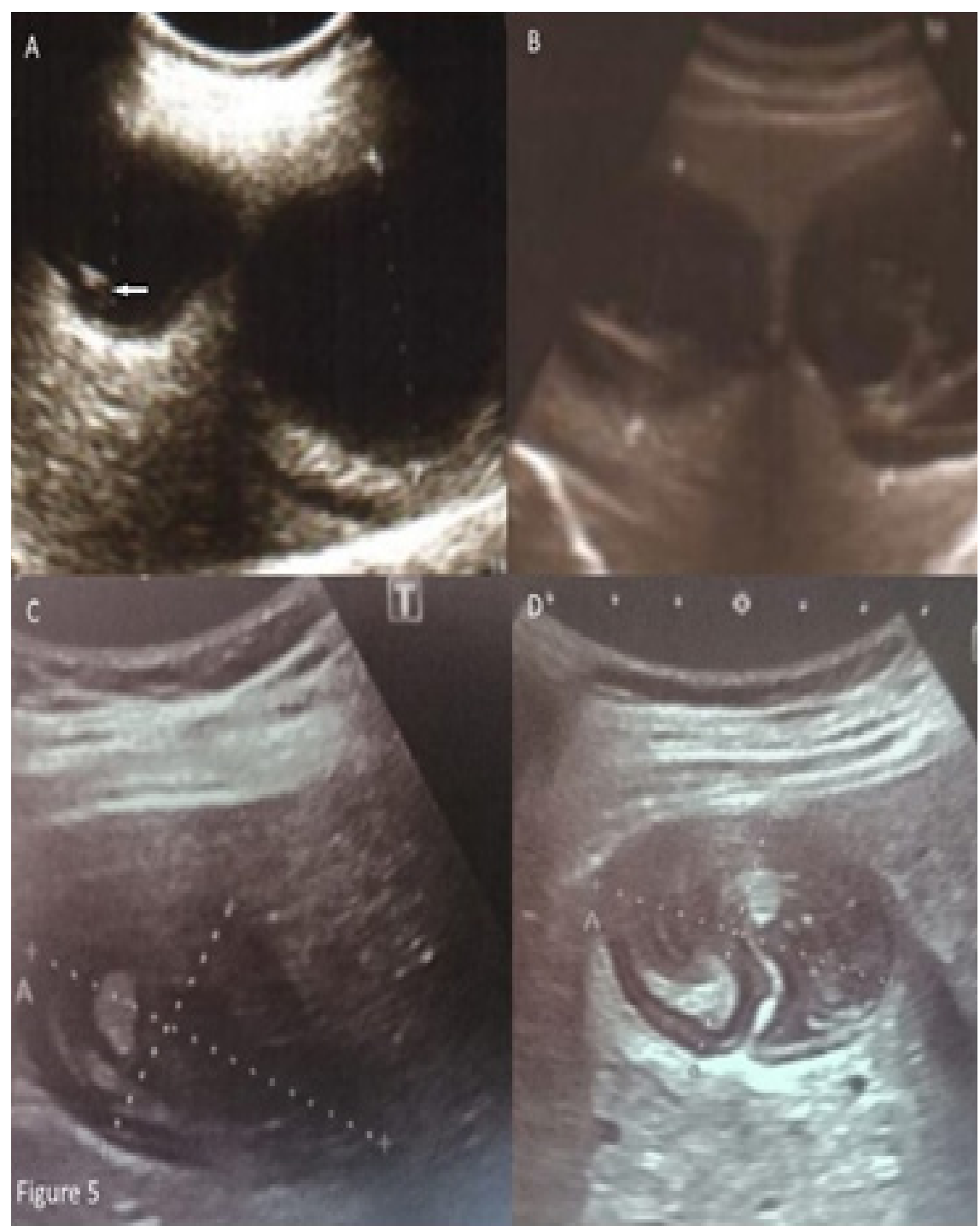

Figure 5 The effect of treatment on cyst that is not directly intervened.

(A) Shows that only one of the cysts was intervened (arrow: needle tip).

(B) Although the second cyst was not intervened, it was seen that both cysts were degenerated after treatment. In the follow-up.

(C) and (D), both cysts appear to be inactivated and solidified.

(D) There is a 'ball of wool' sign.

Table I Baseline Characteristics of the Patients with Hydatid Cysts

\begin{tabular}{ll}
\hline & $\mathbf{n}(\%)$ \\
\hline Number of Patients & 474 \\
Number of HC & 537 \\
Gender(Female-Male) & $303(63.92)-$ I7I (36.07) \\
Type I HC & $270(50.27)$ \\
Type 2 HC & $61(11.35)$ \\
Type 3 HC & $206(38.36)$ \\
& Mean \pm Standart Deviation \\
Age & $43.88 \pm 16.07$ \\
Diamater & $83.34 \pm 34.62$ \\
Follow-up Time & $51.24 \pm 62.99$
\end{tabular}

Table 2 Results of Percutaneous Treatment with Ormeci Technique for Hydatid Cysts

\begin{tabular}{ll}
\hline Parameters & Number of the cysts(\%) \\
\hline HC disappearance & $2 \mathrm{I}(3.91 \%)$ \\
Detachment of Germinative Membranes & $25 \mathrm{I}(46.74 \%)$ \\
Decrease in Diameter & $47 \mathrm{I}(87.70 \%)$ \\
Pseudo-solidification & $489(91.06 \%)$ \\
Calcification & $83(15.45 \%)$ \\
Minimal Changes & $4(0.74 \%)$ \\
\hline
\end{tabular}


Table 3 Complications of the Hydatid Cyst Pre- and After the Treatment

\begin{tabular}{|c|c|c|}
\hline Type of the complications & $\begin{array}{l}\text { Before the } \\
\text { treatment }\end{array}$ & $\begin{array}{l}\text { After the } \\
\text { treatment }\end{array}$ \\
\hline Abcess & 1 & 0 \\
\hline Anaphylaxis & 0 & 3 \\
\hline Increased Liver Enzymes & 7 & 14 \\
\hline Indirect Bilirubinemia & 2 & 10 \\
\hline Uriticeria & I & 2 \\
\hline Direct Bilirubinemia & I & 0 \\
\hline Hypotension & 0 & 3 \\
\hline Fever & 25 & 5 \\
\hline Ruptured into pleura & 2 & 2 \\
\hline Ruptured into bile duct & 7 & 3 \\
\hline Fistulized to the skin & I & 0 \\
\hline Opened to peritonium & 1 & 0 \\
\hline Compression to the portal vein & 2 & 0 \\
\hline Bleed into the cyst & I & 0 \\
\hline
\end{tabular}

\section{Discussion}

Hydatid disease is endemically seen in some areas such as east Europe, Middle East, Australia and New Zealand in which the sheep and cow are growing up. It becomes a heavy economic burden on the people by costing 285,407 Disability Adjusted Life Years (DALYs) or approximately $193,529.740$ US Dollars annually in that countries.

Although hydatid disease is a well-known illness since Hippocrates, the treatment of CE is not optimal yet. Treatment of CE type 4 and 5 cysts are not necessary because of death and keeping the stability for a long time. The asymptomatic cysts smaller than $3-4 \mathrm{~cm}$ in diameter can be followed up until they become symptomatic in the watch and wait strategy. All cysts which are symptomatic, complicated or uncomplicated should be treated in the most convenient manner for each patient.

Benzimidazole compounds (Albendazole (ABZ) and Mebendazole (MBZ) were approved as anthelminthic drugs in 1967. They inhibit beta tubulin's polymerization which is cytoskeletal protein into microtubules and decrease the effect of glucose uptake. Lastly, they cause depletion of glycogen storage and degenerative changes in the endoplasmic reticulum and mitochondria of the germinative membrane of the cyst and results in cellular autolysis. ${ }^{2}$ A treatment using only Benzimidazole carbamates for $\mathrm{CE}$ is not an ideal strategy. Stojkovic et al. ${ }^{3}$ reported that $50-75 \%$ for CE type $1,30-55 \%$ for CE type 2 and $3 \mathrm{~B}$ of $1308 \mathrm{HC}$ which were treated using Benzimidazole derivatives for one and/or two years were inactivated. However, $40 \%$ of those cysts were activated in two years after the treatment. ${ }^{3}$

Franchi et al. ${ }^{4}$ reported that 929 hydatid cysts were treated with Benzimidazole compounds for three to six months. Of the cysts, $75 \%$ degenerated, however, approximately $25 \%$ recurred after $1-14$ years of follow up. ${ }^{4}$ In this randomized clinical study, Albendazole was found to be more effective $(82.2 \%$ versus $56.1 \%$; $\mathrm{P}<0.001)$ than Mebendazole in terms of degenerative changes. In another study by Bari et al. ${ }^{5}$ which compared the patients (Group A) who took ABZ $10 \mathrm{mg} / \mathrm{Kg}$ for three months preoperatively and three months post- operatively and the patients (Group B) who were directly operated for hydatid cyst. Pre-operative examinations showed that all cysts' scolex were viable in group B while $5.5 \%$ of cysts' scolex were viable and there was no recurrence in group B after five years follow-up. ${ }^{5}$ Similarly, treatment outcomes were better when PAIR combined with pre and post-procedure ABZ therapy $10 \mathrm{mg} / \mathrm{kg} / \mathrm{day}$, for three months in the meta-analysis studies conducted by Valesco-Tirado et al. ${ }^{6}$

Surgery is still the mainstay treatment of complicated hydatid cysts. The type of surgery (laparoscopic, radical or conservative treatment) can be determined according to patients' age, co-morbid diseases, size; number, location of the cysts, experience of surgeons and back-ground such as the equipment of the surgical department. Operation time and the hospital stay of laparoscopic surgery are shorter than opened surgical treatment and it can especially be preferred in uncomplicated cysts located peripherally in the anterior segment of the liver. ${ }^{7}$ However, open surgery should be chosen in the multivesiculated, complicated and/or calcified cysts located deeply and near to vena cava inferior. ${ }^{7}$ Radical surgery has fewer complications such as fistulae and cavity infection compared to the conservative one. However, the elderly patients who have co-morbid diseases and cysts that are complicated and larger than $10 \mathrm{~cm}$ in diameter should be treated with the conservative surgical method. ${ }^{8}$ Omentoplasty may be preferred to decrease deep abdominal complications in patients with preoperative complications such as fistula, thick peri-cyst and more than three cysts tube drainage. ${ }^{9}$ In a meta-analysis, 1028 patients with $\mathrm{HC}$ were treated with open surgery $(\mathrm{n}=816)$ or the laparoscopic method $(n=212)$. There were no statistical differences in terms of complications, recurrences, mortality; morbidity and cure rates between the two techniques. ${ }^{7}$ In another meta-analysis comparing laparoscopic surgery and PAIR technique for the treatment of hydatid cyst, PAIR was found to be better in terms of cure of the disease, fewer complications and mortality but it was also inferior in terms of recurrence. $^{10}$

Ben Amor et al. punctured the hydatid cysts in 20 adult sheep and successfully treated $70 \%$ of 30 cysts in $1986 .{ }^{11}$ After this experience, many centers in the world applied the puncture method to cysts, aspiration of the cyst juice, injection of scolicidal agents such as hypertonic saline solutions and re-aspiration of the cyst juice was named as PAIR technique. Today, it is widely accepted that the PAIR technique is effective and safe with less mortality and morbidity rate, cost, complications, hospital stay, and high cure rate compared to surgical methods and treatments..$^{10,12-14}$ Pre and postprocedural combination of ABZ together with PAIR is more effective and has less recurrence rate compare to PAIR alone. ${ }^{15,16}$ Different percutaneous techniques such as the double puncture aspiration injection (DPAI) technique, simple thin catheter, modified catheter technique (MoCAT); special cutting device, percutaneous evacuation technique were used in cysts larger than $5 \mathrm{~cm}$ in diameter or the multivesiculated and/or complicated cysts. However, those techniques have some disadvantages and more complications such as cystobiliary fistula, cavity infection and longer hospital stay. ${ }^{17-19}$ Also, there are no randomized clinical trials comparing those techniques except PAIR/simple catheter use with surgical treatments. Eightyeight patients with $106 \mathrm{HC}$ were treated by comparing two techniques (the Seldinger and catheter techniques) in terms of effectiveness, early complications, local recurrences and secondary dissemination. There were no differences among that techniques. ${ }^{20}$

To our best knowledge, the present study is the largest case series for the percutaneous treatment of hydatic cyst in a single center. 
Unlike in the PAIR method, the aspiration of a small amount of cyst fluid varying between 12-60 cc can cause small a decrease in the inside pressure of cysts in the Ormeci technique. This is the cause of having less cysto-biliary fistulae. Predictive factors for biliary fistulae were jaundice, increasing of liver enzymes and size of the cysts. ${ }^{21}$ The amount of juice aspirated from the cyst cavity and intracystic pressure should be considered among the other risk factors. The incidence of biliary fistula varied between $2-75 \%$ in different series. ${ }^{22}$ Saremi and McNamara reported that the fistulae rate by PAIR technique was $34.3 \%$ in their case series. This could be the reason for the decrease in the pressure of cysts. ${ }^{19} \mathrm{We}$ did not have any case of fistulae in our series.

We did not use catheters even in the cysts larger than $23 \mathrm{~cm}$ in diameter. This decreased the possibility of infection. Developing cavity infection or abscess were reported to be between 5.6\%-26.9\% in the literature. ${ }^{18-23}$

There are several advantages of the Ormeci technique over the PAIR(18) (Table 4). We did not have any patients who had abscess or infection in the cyst cavity. Using a thinner needle may decrease the leakage of cyst fluid. We did not observe any complications due to puncture with multiple needles at the same time of the procedure. There was no mortality in this study. Three patients $(0.63 \%)$ had non-fatal anaphylaxis. They were treated medically and all of them survived. We had totally 42 non-fatal complications among 474 patients. In their meta-analysis, Neumayr et al. ${ }^{24}$ reported that 5517 patients who had percutaneous treatment procedures had two $(0.03 \%)$ lethal and $99(1.7 \%)$ reversible anaphylactic reactions. ${ }^{24}$

Table 4 Main Therapeutic Differences between PAIR and Ormeci Techniques

\begin{tabular}{lll}
\hline & PAIR & Ormeci \\
\hline Needle Type & I8-20 Gauge & 22 Gauge \\
$\begin{array}{lll}\text { Amount of Fluid } \\
\text { Aspiration }\end{array}$ & I/3-I/2 of cyst volume & I2-60cc \\
Sclerosing Agent & $\begin{array}{l}\text { Pure alcohol or } \\
\text { hypertonic saline }\end{array}$ & $\begin{array}{l}\text { Pure Alcohol plus } \\
\text { Polydocanol }\end{array}$ \\
$\begin{array}{l}\text { Catheter } \\
\text { Time Period }\end{array}$ & $\begin{array}{l}\text { Until the drainage stops } \\
\text { spontaneously }\end{array}$ & 5 minutes \\
$\begin{array}{l}\text { Length of } \\
\text { Hospital Stay }\end{array}$ & Long & Outheter \\
Chemotherapy & $\begin{array}{l}\text { Albendazole or } \\
\text { Praziquantel }\end{array}$ & No Chemotherapy \\
Re-treatment & Rare & As needed
\end{tabular}

Valesco-Tirado et al. ${ }^{25}$ reported that $19 \%$ of 193 patients who used Albendazole had adverse events. ${ }^{25}$ We did not use chemotherapy before or after the procedure. This decreased the morbidity of the Ormeci technique. One of the important advantages of this technique was that it was repeatable. CE type 2 and $3 \mathrm{~B}$ cysts were difficult to treat at one puncture. Multi puncture of the cyst for different localizations at the same session and dividing the sclerosing agents into different locations increased the cure rate of CE 2 and $3 \mathrm{~B}$ (Gharbi type 3 ). The cure rate of type CE 2 and type $3 \mathrm{~B}$ or (Gharbi type 3 ) was $91.7 \%$ in our case series. However, Akhan et al. ${ }^{18}$ Giorgio et al. ${ }^{23}$ and Kabaalioglu et al ${ }^{27}$ reported the cure rate of multi-vesiculated hydatid cysts to be $41.4 \%, 52.2 \%$ and $61.5 \%$; respectively. ${ }^{18,26,27} \mathrm{CE}$ type 1 and $3 \mathrm{~A}$ can be successfully treated with 3.60 fold and 3.82 fold compared to CE type 2 and 3B hydatid cyst, respectively $(\mathrm{p}<0.001, \% 95 \mathrm{CI}$ : 2.13-6.07 and $\mathrm{p}=0.006,95 \% \mathrm{CI}: 1.47-9.89$ ).

We did not have a case that had sclerosing cholangitis after $51.17 \pm 2.70$ months of follow-up. The reason for this should be the use of a small amount of sclerosing agents. Twenty HC (3.9\%) disappeared after the treatment. This varied between $4.7-81 \%$ in other series. ${ }^{23,28}$ Less disappearance of the $\mathrm{HC}$ in our series should be caused by the small amount of aspiration from the cyst cavity. A small amount of aspiration of cysts' fluid protects the occurrence of fistulae because of the small difference of pressure between the bile ducts. The cure rate of this series was $95 \%$. Nayman et al. ${ }^{29}$ treated 374 patients with 493 hydatid cysts by six French catheters. They reported a 97.7\% cure rate. ${ }^{29}$ Cure rates of PAIR technique vary between $80 \%-99.5 \%$ in the literature. ${ }^{27}$

One of the weak points of this study was the irregular follow-up time of the patients whom we sometimes had to evaluate using the ultrasonographic pictures which were taken in their city.

\section{Conclusions}

This is the largest series for percutaneous treatment using the Ormeci technique of Hydatid cyst in a single center. It is more successful in CE type 2 and 3B hydatid cysts. This technique has no mortality and less morbidity, easy to perform, cost-effective and repeatable. Compare to PAIR techniques and surgical treatment, It should be used for the treatment in all active stages of the hydatid cysts.

\section{Conflicts of interest}

All authors declared there are no conflicts of interest.

\section{Acknowledgments}

My coauthors and I were thankful to Kadir Daştan, M.D. for revision of this manuscript.

\section{Funding}

None.

\section{References}

1. Budke CM, Deplazes P, Torgerson PR. Global socioeconomic impact of cystic echinococcosis. Emerg Infect Dis. 2006;12(2):296-303.

2. Dehkordi AB, Sanei B, Yousefi M, et al. Albendazole and Treatment of Hydatid Cyst: Review of the Literature. Infect Disord Drug Targets. 2019;19(2):101-104.

3. Stojkovic M, Zwahlen M, Teggi A, et al. Treatment response of cystic echinococcosis to benzimidazoles: a systematic review. PLoS Negl Trop Dis. 2009;3(9):e524-e.

4. Franchi C, Di Vico B, Teggi A. Long-term evaluation of patients with hydatidosis treated with benzimidazole carbamates. Clin Infect Dis. 1999;29(2):304-309.

5. Arif S, Malik A, Khaja A, et al. Role of albendazole in the management of hydatid cyst liver. Saudi Journal of Gastroenterology. 2011;17(5):343347.

6. Velasco-Tirado V, Alonso-Sardón M, Lopez-Bernus A, et al. Medical treatment of cystic echinococcosis: systematic review and meta-analysis. BMC Infect Dis. 2018;18(1):306.

7. Sokouti M, Sadeghi R, Pashazadeh S, et al. A systematic review and metaanalysis on the treatment of liver hydatid cyst: Comparing laparoscopic and open surgeries. Arab J Gastroenterol. 2017;18(3):127-135. 
8. Gupta N, Javed A, Puri S, et al. Hepatic hydatid: PAIR, drain or resect? J Gastrointest Surg. 2011;15(10):1829-1836.

9. Dziri C, Paquet JC, Hay JM, et al. Omentoplasty in the prevention of deep abdominal complications after surgery for hydatid disease of the liver: a multicenter, prospective, randomized trial. French Associations for Surgical Research. J Am Coll Surg. 1999;188(3):281-289.

10. Sokouti M, Sadeghi R, Pashazadeh S, et al. A systematic review and meta-analysis on the treatment of liver hydatid cyst using meta-MUMS tool: comparing PAIR and laparoscopic procedures. Arch Med Sci. 2019;15(2):284-308.

11. Ben Amor N, Gargouri M, Gharbi HA, et al. Treatment of hepatic hydatid cyst in sheep by echographic puncture. Tunis Med. 1986;64(4):325-331.

12. Smego RA Jr, Bhatti S, Khaliq AA, et al. Percutaneous aspirationinjection-reaspiration drainage plus albendazole or mebendazole for hepatic cystic echinococcosis: a meta-analysis. Clin Infect Dis. 2003;37(8):1073-1083.

13. Shera TA, Choh NA, Gojwari TA, et al. A comparison of imaging guided double percutaneous aspiration injection and surgery in the treatment of cystic echinococcosis of liver. Br J Radiol. 2017;90(1072):20160640.

14. Dziri C, Haouet K, Fingerhut A. Treatment of hydatid cyst of the liver: where is the evidence? World J Surg. 2004;28(8):731-736.

15. Akhan O, Yildiz AE, Akinci D, et al. Is the adjuvant albendazole treatment really needed with PAIR in the management of liver hydatid cysts? A prospective, randomized trial with short-term follow-up results. Cardiovasc Intervent Radiol. 2014;37(6):1568-1574.

16. Falagas ME, Bliziotis IA. Albendazole for the treatment of human echinococcosis: a review of comparative clinical trials. Am J Med Sci. 2007;334(3):171-179.

17. Schipper HG, Laméris JS, van Delden OM, et al. Percutaneous evacuation (PEVAC) of multivesicular echinococcal cysts with or without cystobiliary fistulas which contain non-drainable material: first results of a modified PAIR method. Gut. 2002;50(5):718-723.

18. Akhan O, Salik AE, Ciftci T, et al. Comparison of Long-Term Results of Percutaneous Treatment Techniques for Hepatic Cystic Echinococcosis Types 2 and 3b. AJR Am J Roentgenol. 2017;208(4):878-884.
19. Saremi F, McNamara TO. Hydatid cysts of the liver: long-term results of percutaneous treatment using a cutting instrument. AJR Am J Roentgenol. 1995;165(5):1163-1167.

20. Turan HG, Özdemir M, Acu R, et al. Comparison of seldinger and trocar techniques in the percutaneous treatment of hydatid cysts. World J Radiol. 2017;9(11):405-412.

21. El Nakeeb A, Salem A, El Sorogy M, et al. Cystobiliary communication in hepatic hydatid cyst: predictors and outcome. Turk J Gastroenterol. 2017;28(2):125-130.

22. Akkapulu N, Aytac HO, Arer IM, et al. Incidence and risk factors of biliary fistulation from a hepatic hydatid cyst in clinically asymptomatic patients. Trop Doct. 2017;48(1):20-24.

23. Giorgio A, de Stefano G, Esposito V, et al. Long-term results of percutaneous treatment of hydatid liver cysts: a single center 17 years experience. Infection. 2008;36(3):256-261.

24. Neumayr A, Troia G, de Bernardis C, et al. Justified concern or exaggerated fear: the risk of anaphylaxis in percutaneous treatment of cystic echinococcosis-a systematic literature review. PLoS Negl Trop Dis. 2011;5(6):e1154.

25. Velasco-Tirado V, Romero-Alegria A, Pardo-Lledías J, et al. Management of cystic echinococcosis in the last two decades: what have we learned? Trans R Soc Trop Med Hyg. 2018;112(5):207-215.

26. Giorgio A, Di Sarno A, de Stefano G, et al. Sonography and clinical outcome of viable hydatid liver cysts treated with double percutaneous aspiration and ethanol injection as first-line therapy: efficacy and longterm follow-up. AJR Am J Roentgenol. 2009;193(3):W186-W192.

27. Kabaalioğlu A, Ceken K, Alimoglu E, et al. Percutaneous imaging-guided treatment of hydatid liver cysts: do long-term results make it a first choice? Eur J Radiol. 2006;59(1):65-73.

28. Golemanov B, Grigorov N, Mitova R, et al. Efficacy and safety of PAIR for cystic echinococcosis: experience on a large series of patients from Bulgaria. The American journal of Tropical Medicine and Hygiene. 2011;84(1):48-51.

29. Nayman A, Guler I, Keskin S, et al. A novel modified PAIR technique using a trocar catheter for percutaneous treatment of liver hydatid cysts: a six-year experience. Diagn Interv Radiol. 2016;22(1):47-51. 